\title{
Lack of mutations of $G 4.5$ in three families from China with noncompaction of the ventricular myocardium
}

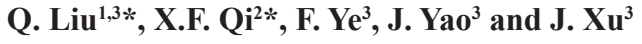 \\ ${ }^{1}$ Department of Gastroenterology, First Affiliated Hospital, \\ Soochow University, Suzhou, China \\ ${ }^{2}$ Leukemia Research Division, Jiangsu Institute of Hematology, \\ First Affiliated Hospital, Soochow University, Suzhou, China \\ ${ }^{3}$ Department of Cardiology, Nanjing First Hospital, Nanjing, China \\ *These authors contributed equally to this study. \\ Corresponding author: J. Xu \\ E-mail: drxujing@126.com
}

Genet. Mol. Res. 12 (1): 53-58 (2013)

Received February 8, 2012

Accepted July 30, 2012

Published January 22, 2013

DOI http://dx.doi.org/10.4238/2013.January.22.3

\begin{abstract}
To find the underlying cause of noncompaction of the ventricular myocardium (NVM), three Chinese families with probands who presented this problem were studied. After the family members were evaluated by echocardiography, the gene G4.5 (taffazin) was scanned by sequencing. Although X-linked inheritance could not be ruled out, NVM were thought to have a vague rule of inheritance in our data from 8 patients and 28 family members. We also did not identify any mutations in $G 4.5$ in all samples. Our data suggest that other genes are responsible for the familial form of this disease.
\end{abstract}

Key words: Noncompaction of the ventricular myocardium; $G 4.5$; Mutations 


\section{INTRODUCTION}

Noncompaction of the ventricular myocardium (NVM) is a rare congenital cardiomyopathy (Jenni et al., 2001) that results from abnormal arrest in endomyocardial embryogenesis (Weiford et al., 2004) and is characterized by the presence of prominent ventricular myocardial trabeculations and deep intertrabecular recesses, while the compacted myocardium is thin (Zambrano et al., 2002; Maron et al., 2006; Klaassen et al., 2008). Some studies have showed that NVM is an autosomal dominant inherited disease (Sasse-Klaassen et al., 2003) and mutations in the $G 4.5$ gene (taffazin) on the Xq28 chromosomal region may be responsible for some familial cases (Chin et al., 1990; Ichida et al., 2001; Chen et al., 2002; Kenton et al., 2004). However, there are no genetic research results on NVM in China.

\section{MATERIAL AND METHODS}

\section{Clinical evaluation}

Three families from China were recruited for this study. Probands and available family members were evaluated by medical record review, physical examination and history taking, 24-h Holter monitoring, 12-lead electrocardiography, and echocardiography. All electrocardiography results were performed and reviewed by 2 or more independent observers according to institutional guidelines. The criteria for diagnosis were 1) the presence of prominent trabeculations with deep intertrabecular recesses; 2) the noncompacted endocardial layer was thicker than the compacted epicardial layer (ratio $\geq 2$ ), and 3 ) the presence of deep recesses filled with blood from the ventricular cavity, as visualized by color Doppler imaging.

\section{Mutation screening}

Genomic DNA was isolated from peripheral blood using a Wizard Genomic DNA Purification kit (Takara, China). Primers were designed by Primer3 according to the NCBI database (Table 1). Polymerase chain reaction (PCR) was performed in $50-\mu \mathrm{L}$ reactions containing $2 \mathrm{U}$ Pyrobest DNA Polymerase (Takara), $0.5 \mu \mathrm{M}$ each of forward and reverse primers, and 50 ng genomic DNA in $1 \mathrm{X}$ Pyrobest buffer (containing $\mathrm{MgCl}_{2}$ ). Amplification was performed with an initial step of $94^{\circ} \mathrm{C}$ for $5 \mathrm{~min}$; followed by 30 cycles of $94^{\circ} \mathrm{C}$ for $30 \mathrm{~s}$, annealing for $30 \mathrm{~s}$, and $72^{\circ} \mathrm{C}$ for $30 \mathrm{~s}$, and then $72^{\circ} \mathrm{C}$ for $10 \mathrm{~min}$. PCR products were purified using a Qiagen PCR purification kit and sequenced using an ABI 3730 DNA Genetic Analyzer.

\section{RESULTS}

\section{Study cohort}

Three of 9 probands agreed to an extended pedigree analysis; their 1st-degree relatives were clinically evaluated and echocardiography was performed. The relatives of the remaining 6 probands were unavailable or declined to participate in the study, and we were consequently unable to determine whether these were sporadic or familial cases of NVM. In the 3 kindred evaluated, at least one or another affected relative was identified. Therefore, 
based on this investigation, these 3 probands were considered to have familial NVM (kindred NVM, Figure 1A-C).

\begin{tabular}{|c|c|c|c|c|}
\hline & Sequence & Exon & Products (bp) & $\operatorname{Tm}\left({ }^{\circ} \mathrm{C}\right)$ \\
\hline $1 \mathrm{~F}$ & 5'-CTGACCTGCGAAGGGACCTCGGTC-3' & $1 \sim 2$ & 644 & 61 \\
\hline $1 \mathrm{R}$ & 5'-CCAGGGCTCCATGAAAAGGC-3' & & & \\
\hline $2 \mathrm{~F}$ & 5'-GGCTGTAGGGAAATGGTAGTGCTGC-3' & $3 \sim 4$ & 588 & 62 \\
\hline $2 \mathrm{R}$ & 5'-CCTCATGTTGGCTCCCGGGATGCC-3' & & & \\
\hline $3 \mathrm{~F}$ & 5'-GGCCAGAGGGTTTTGCTTCTAGG-3' & 5 & 259 & 63 \\
\hline $3 \mathrm{R}$ & 5'-CTCCTGGGCTTGAGTGATCCTCTC-3' & & & \\
\hline $4 \mathrm{~F}$ & 5'-GGAGAAGGGCCTGTTTCATTGAG-3' & $6 \sim 7$ & 471 & 62 \\
\hline $4 \mathrm{R}$ & 5'-CGAGAGGCAGGCCTAGTCTCAGC-3' & & & \\
\hline $5 \mathrm{~F}$ & 5'-GGTGGCAGTGGCCAGAGGCTG-3' & $8 \sim 9$ & 386 & 62 \\
\hline $5 \mathrm{R}$ & 5'-ATAGTGCTGAGTGGGAGACACGG-3' & & & \\
\hline $6 \mathrm{~F}$ & 5'-CCTTGTTTTAGAGGAAGAGTGGCC-3' & $10 \sim 11$ & 618 & 58 \\
\hline $6 \mathrm{R}$ & 5'-AGCTCGGAGAGGGCACTTGAG-3' & & & \\
\hline
\end{tabular}

A
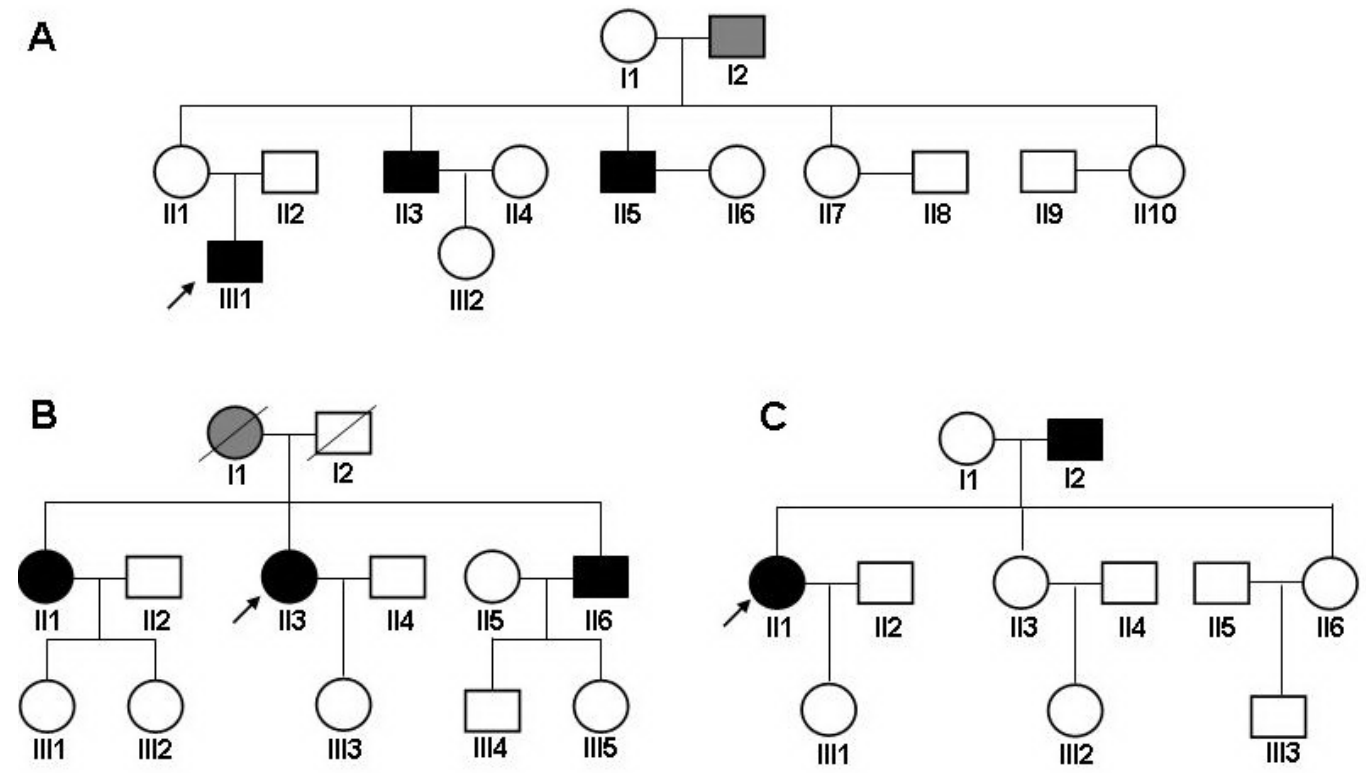

Figure 1. Pedigree of the three families. All sampled subjects are identified by the Roman numerals below the symbol. Arabic numerals denote each individual in a generation. Open symbols = unaffected; filled symbols = affected; symbols with a diagonal line $=$ deceased subjects; squares = males; circles = females; A. family 1 ; $\mathbf{B}$. family 2; C. family 3 .

Kindred NVM-1 was diagnosed in the proband (Figure 1A) when he was 17 years old and experienced atrial fibrillation. The cardiac status of the patient was evaluated. Echocardiography revealed dilatation of the left and right atria and left ventricle with barely normal systolic and abnormal diastolic function (LVEDD, $5.9 \mathrm{~cm}$; LVEF, 56\%) (Figure 2A and B). The patient's PASP, measured from TR, was $110 \mathrm{mmHg}(1 \mathrm{mmHg}=0.133 \mathrm{kPa}) .{ }^{99 \mathrm{~m} T c-M A A}$ 
pulmonary perfusion and ventilation imaging revealed blood flow reduction in the right lower basal segment of the pulmonary artery that could not match normal ventilation, suggesting pulmonary embolism. Three other members of kindred NVM-1 presented either heart failure or arrhythmia symptoms in their 4th decade of life. All of them were male. Individuals I-2 (75 years old), II-3 (45 years old), and II-5 (49 years old) exhibited typical features of noncompaction of the left ventricular apex and posterior wall: prominent left ventricular trabeculations; noncompacted endocardial layer less than 2 times thicker than the compacted epicardial layer.

A

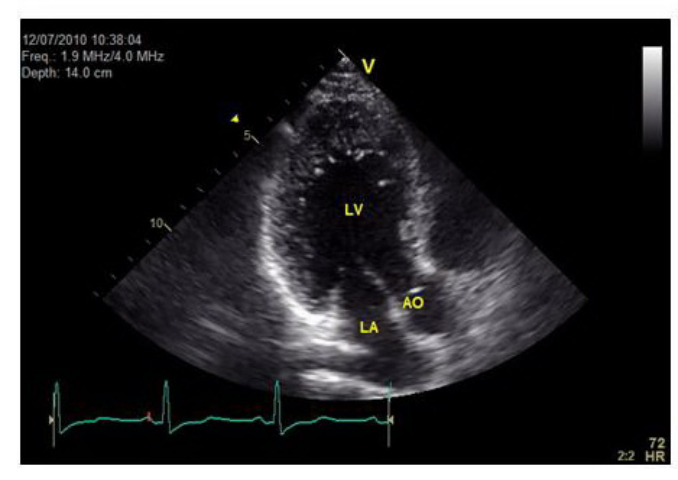

B

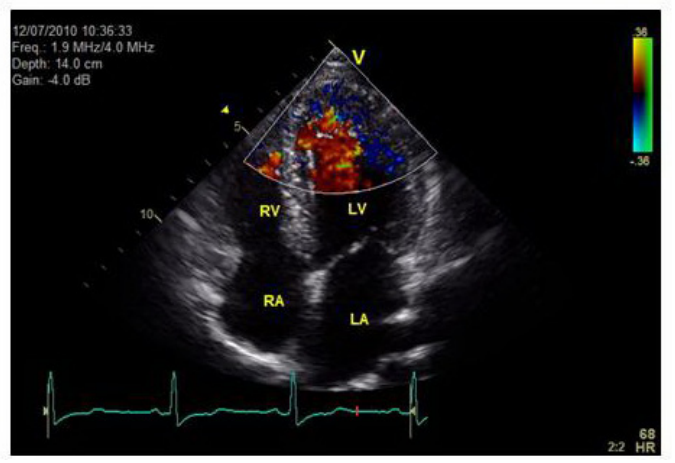

Figure 2. Parasternal long axis view (A) and apical long axis view (B) of a 17-year-old patient. The enddiastolic still frame shows the markedly thickened inferior, septal and lateral wall. Two-layer structure consists of the compacted epicardial layer and the noncompacted endocardial layer. The deep recesses are filled with blood from the ventricular cavity as visualized by color Doppler imaging (B).

In kindred NVM-2 (Figure 1B), the proband (II-3), a 57-year-old woman, presented palpitation and shortness of breath upon admission, and on examination, moisture rales were heard on both sides; in the heart, a 3rd-degree systolic murmur was auscultated. Marked noncompaction confined to the left ventricular apex and enlargement of the left ventricle with reduced systolic function were identified (LVEDD, $6.1 \mathrm{~cm}$; LVEF, 45\%). Holter monitoring revealed a polymorphic ventricular premature beat, paroxysmal atrial tachycardia, and V5 ST depression. The proband's sister and brother, II-1 (61 years old) and II-6 (55 years old), respectively, have been diagnosed with NVM. Thus, from 3 generations (Figure 1B), i.e., of 13 people, 3 individuals, including the proband, were diagnosed with NVM. The proband's siblings both experienced palpitation after exertion. Holter monitoring revealed that II-1 experienced frequent premature ventricular contractions; II-6 was noted to have atrial fibrillation. The history of the proband's mother, I-1, was confirmed: she had heart dysfunction for many years and died suddenly when she was 59 years old; the father, I-2, died of stroke when he was 72 years old, leaving insufficient details about any significant link to NVM.

In kindred NVM-3, the proband (II-1) was a 57-year-old woman. She was admitted after presenting complaints of repeated chest tightness (Figure 1C). On echocardiography, prominent trabeculations with deep intertrabecular recesses were found in the apex and inferior and lateral walls of the left ventricle. The noncompacted endocardial layer was thicker than the compacted epicardial layer (ratio $=2.5$ ). Holter monitoring revealed paroxysmal 2nd-degree type 1 
atrioventricular block. An angiogram revealed a normal coronary system. In this family, which spanned 3 generations (11 individuals), none but the proband was found to be affected with NVM. The proband's 72-year-old father, I-2, was diagnosed with hypertrophic cardiomyopathy when he was 68 years old; echocardiography and clinical symptoms supported the diagnosis.

\section{G4.5 mutation analysis}

Although X-linked inheritance could not be ruled out, NVM was thought to adhere to a vague rule of inheritance in these cases. Although mutations of the $G 4.5$ gene were thought to be responsible for some familial cases of NVM, no mutants in either the probands or other samples were found in our research.

\section{DISCUSSION}

NVM is categorized as an unclassified cardiomyopathy in the World Health Organization Task Force report (Richardson et al., 1996). The prevalence is approximately $0.014 \%$ (Kasifoglu et al., 2007). Transthoracic echocardiography, transesophageal echocardiography, and magnetic resonance imaging are used to diagnose NVM.

On the basis of the data for the responsible G4.5 gene, Bleyl et al. (1997) proposed that NVM was an X-linked inherited disease. Ichida et al. (2001) noted that the autosomal dominant inheritance pattern also existed in NVM patients. Our results showed that although $\mathrm{X}$-linked inheritance could not be ruled out, NVM in our cases was thought to adhere to a vague rule of inheritance. As in other heart muscle disorders, NVM demonstrates genetic heterogeneity. Mutations in genes encoding G4.5 (taffazin) have been reported as causes of the disease in several reports (Chin et al., 1990; Ichida et al., 2001; Chen et al., 2002), but there are patients in whom no genetic basis of disease is found to be significant. Some studies reported that G4.5 mutations are rare in familial cases of NVM (Chen et al., 2002; Sasse-Klaassen et al., 2003; Kenton et al., 2004). Klaassen et al. (2008) identified no mutations in 25 probands. In the study performed by Chen et al. (2002) mutations were identified in only 1 patient from 27 Japanese subjects. Our results also did not show G4.5 mutations in any of the subjects. Our data suggest that other genes are responsible for this disease, such as $\alpha$-dystrobrevin, $R y R 2$, MYH7, ACTC, and TNNT2 (Klaassen et al., 2008).

\section{ACKNOWLEDGMENTS}

Research supported by the National Natural Scientific Foundation of China (Grant \#81170468), the Natural Scientific Foundation of Jiangsu (Grant \#BK2011266), and the Nanjing Medical Scientific Foundation (Grant \#YKK08094).

\section{REFERENCES}

Bleyl SB, Mumford BR, Thompson V, Carey JC, et al. (1997). Neonatal, lethal noncompaction of the left ventricular myocardium is allelic with Barth syndrome. Am. J. Hum. Genet. 61: 868-872.

Chen R, Tsuji T, Ichida F, Bowles KR, et al. (2002). Mutation analysis of the G4.5 gene in patients with isolated left ventricular noncompaction. Mol. Genet. Metab. 77: 319-325.

Chin TK, Perloff JK, Williams RG, Jue K, et al. (1990). Isolated noncompaction of left ventricular myocardium. A study 
of eight cases. Circulation 82: 507-513.

Ichida F, Tsubata S, Bowles KR, Haneda N, et al. (2001). Novel gene mutations in patients with left ventricular noncompaction or Barth syndrome. Circulation 103: 1256-1263.

Jenni R, Oechslin E, Schneider J, Attenhofer JC, et al. (2001). Echocardiographic and pathoanatomical characteristics of isolated left ventricular non-compaction: a step towards classification as a distinct cardiomyopathy. Heart 86: 666-671.

Kasifoglu T, Cavusoglu Y, Korkmaz C and Birdane A (2007). Noncompaction of the ventricular myocardium in a patient with Behcet's disease showing multiple thrombus formations in the right atrium, inferior vena cava and right iliac vein: A case report. Int. J. Angiol. 16: 69-72.

Kenton AB, Sanchez X, Coveler KJ, Makar KA, et al. (2004). Isolated left ventricular noncompaction is rarely caused by mutations in G4.5, alpha-dystrobrevin and FK Binding Protein-12. Mol. Genet. Metab. 82: 162-166.

Klaassen S, Probst S, Oechslin E, Gerull B, et al. (2008). Mutations in sarcomere protein genes in left ventricular noncompaction. Circulation 117: 2893-2901.

Maron BJ, Towbin JA, Thiene G, Antzelevitch C, et al. (2006). Contemporary definitions and classification of the cardiomyopathies: an American Heart Association Scientific Statement from the Council on Clinical Cardiology, Heart Failure and Transplantation Committee; Quality of Care and Outcomes Research and Functional Genomics and Translational Biology Interdisciplinary Working Groups; and Council on Epidemiology and Prevention. Circulation 113: $1807-1816$.

Richardson P, McKenna W, Bristow M, Maisch B, et al. (1996). Report of the 1995 World Health Organization/International Society and Federation of Cardiology Task Force on the Definition and Classification of cardiomyopathies. Circulation 93: 841-842.

Sasse-Klaassen S, Gerull B, Oechslin E, Jenni R, et al. (2003). Isolated noncompaction of the left ventricular myocardium in the adult is an autosomal dominant disorder in the majority of patients. Am. J. Med. Genet. A 119A: 162-167.

Weiford BC, Subbarao VD and Mulhern KM (2004). Noncompaction of the ventricular myocardium. Circulation 109: 2965-2971.

Zambrano E, Marshalko SJ, Jaffe CC and Hui P (2002). Isolated noncompaction of the ventricular myocardium: clinical and molecular aspects of a rare cardiomyopathy. Lab Invest 82: 117-122. 